\section{Sur un système d'inégalités intégrales}

\author{
par Z. OPIAL (Kraków)
}

Nous allons considérer le système d'équations différentielles

$$
y_{i}^{\prime}=f_{i}\left(x, y_{1}, \ldots, y_{n}\right) \quad(i=1,2, \ldots, n)
$$

dont les seconds membres sont définis et continus dans un domaine ouvert $\Omega$ de variables $x, y_{1}, \ldots, y_{n}$. Les théorèmes que nous démontrons dans cette note établissent une certaine relation d'inégalité entre les intégrales du système (1) et les fonctions continues $p_{1}(x), \ldots, \varphi_{n}(x)$ satisfaisant au système d'inégalités intégrales

(2) $\quad \varphi_{i}(x) \leqslant \eta_{i}+\int_{\xi}^{x} f_{i}\left(x, \varphi_{1}(x), \ldots, \varphi_{n}(x)\right) d x \quad(i=1,2, \ldots, n$;

$$
\xi \leqslant x \leqslant \xi+\alpha)
$$

où le point $\left(\xi, \eta_{1}, \ldots, \eta_{n}\right)$ appartient au domaine $\Omega$ et $\alpha$ est une constante positive.

Le théorème $I$ donne une telle relation dans l'hypothèse de la croissance des fonctions $f_{i}\left(x, y_{1}, \ldots, y_{n}\right)(i=1, \ldots, n)$ par rapport aux variables $\left.y_{1}, y_{2}, \ldots, y_{n}{ }^{1}\right)$. Il se pose le problème de savoir dans quelle mesure cette hypothèse de la croissance est essentielle pour la validité de ce théorème. Le théorème II constitue la réponse à ce problème, qui a été posé par M. T. Ważewski.

Dans le cas de l'équation $y^{\prime}=f(x, y)$ (c'est-à-dire pour $n=1$ ) le théorème I a été établi par B. Viswanatham [2]. La démonstration de B. Viswanatham s'appuie sur la méthode des approximations successives. La nôtre est fondée sur un autre principe.

§ 1. ThínORऐMe I. Si les fonctions $f_{i}\left(x, y_{1}, \ldots, y_{n}\right) \quad(i=1,2, \ldots, n)$ sont croissantes au sens large par rapport dे l'ensemble des variables $y_{1}, y_{2}, \ldots, y_{n}$, les fonctions $\varphi_{1}(x), \ldots, \varphi_{n}(x)$ continues satisfaisant au système d'inégatités (2) satisfont aux inégalités

$$
\text { (3) } \quad \varphi_{i}(x) \leqslant \psi_{i}(x) \quad(\xi \leqslant x, i=1,2, \ldots, n)
$$

1) Cela veut dire que pour $\bar{y}_{1} \leqslant y_{1}, \ldots, \bar{y}_{n} \leqslant y_{n}$ on a $f_{i}\left(x, \bar{y}_{1}, \ldots, y_{n}\right) \leqslant f_{i}\left(x, y_{1}\right.$, $\left.\ldots, y_{n}\right)(i=1,2, \ldots, n)$ où nous avons désigné par $\psi_{1}(x), \ldots, \psi_{n}(x)$ l'intégrale supérieure à droite du système (1) issue du point $\left.\left(\xi, \eta_{1}, \ldots, \eta_{n}\right)^{2}\right)$. Les inégalités sont vraies $\grave{a}$ droite de $\xi$ dans l'intervalle d'existence de toutes les fonctions qui y interviennent.

Démonstration. En désignant par $\psi_{1}(x, \varepsilon), \psi_{2}(x, \varepsilon), \ldots, \psi_{n}(x, \varepsilon)$ $(\varepsilon>0)$ l'intégrale supérieure à droite du système (1) issue du point $\left(\xi, \eta_{1}+\varepsilon, \ldots, \eta_{n}+\varepsilon\right)$ on a:

(4) $\quad \psi_{i}(x, \varepsilon)=\eta_{i}+\varepsilon+\int_{\xi}^{x} f_{i}\left(x, \psi_{1}(x, \varepsilon), \ldots, \psi_{n}(x, \varepsilon)\right) d x \quad(i=1,2, \ldots, n)$.

Des inégalités (2) et des identités (4) on obtient par soustraction membre à membre

(5) $\quad \varphi_{i}(x)-\psi_{i}(x, \varepsilon) \leqslant-\varepsilon+\int_{\xi}^{x}\left[f_{i}\left(x, \varphi_{1}(x), \ldots\right)-f_{i}\left(x, \psi_{1}(x, \varepsilon), \ldots\right)\right] d x$

d'où, en particulier

(6) $\quad \varphi_{i}(\xi)-\psi_{i}(\xi, \varepsilon) \leqslant-\varepsilon<0 \quad(i=1,2, \ldots, n)$.

Nous allons démontrer que

(7) $\varphi_{i}(x)-\psi_{i}(x, \varepsilon)<0$ pour $\xi<x \quad$ et $i=1,2, \ldots, n$.

Admettons le contraire. Il existe évidemment un $x_{0}>\xi$ tel que pour un certain indice

$$
\varphi_{k}\left(x_{0}\right)-\psi_{k}\left(x_{0}, \varepsilon\right)=0
$$

et que les inégalités ( 7$)$ ont lieu dans l'intervalle $\left\langle\xi, x_{0}\right)$ tout entier. Par suite, on a dans cet intervalle, en vertu de la croissance de la fonction $f_{k}\left(x, y_{1}, \ldots, y_{n}\right)$,

$$
f_{k}\left(x, \varphi_{1}(x), \ldots, \varphi_{n}(x)\right) \leqslant f_{k}\left(x, \psi_{1}(x, \varepsilon), \ldots, \psi_{n}(x, \varepsilon)\right)
$$

d'où, en posant dans (5) $x=x_{0}$ on obtient

$$
\varphi_{k}\left(x_{0}\right)-\psi_{k}\left(x_{0}, \varepsilon\right) \leqslant-\varepsilon+\int_{\xi}^{x_{0}}\left[f_{k}\left(x, \varphi_{1}(x), \ldots\right)-f_{k}\left(x, \psi_{1}(x, \varepsilon), \ldots\right)\right] d x
$$

et par suite $\varphi_{k}\left(x_{0}\right)-\psi_{k}\left(x_{0}, \varepsilon\right) \leqslant-\varepsilon$ ce qui est impossible en raison de la relation (8).

Les inégalités (7) ont done lieu à droite de $\xi$ dans l'intervalle d'existence des fonctions $\varphi_{1}(x), \ldots, \psi_{1}(x, \varepsilon), \ldots ; \varepsilon$ tendant vers zéro, les fonctions $\psi_{i}(x, \varepsilon)$ tendent vers $\psi_{i}(x)$ (voir [1], Satz 10, p. 83) et c'est

2) L'existence de cette intégrale résulte des hypothèses de notre théorème (Ważewski [3]). 
ainsi qu'à partir des inégalités (7) on obtiendra les inégalités (3). Elles auront lieu dans l'intervalle d'existence des fonctions $p_{1}(x), \ldots, \psi_{1}(x), \ldots$ ce qui résulte du théorème suivant:

Pour tout intervalle contenu dans l'intervalle d'existence de l'intégrale $\psi_{1}(x), \ldots, \psi_{n}(x)$ on peut ohoisir un $\bar{\varepsilon}>0$ tel que pour $0<\varepsilon<\bar{\varepsilon}$ toutes les intégrales $\psi_{1}(x, \varepsilon), \ldots, \psi_{n}(x, \varepsilon)$ y soient définies (voir [1], Satz 10, p. 83).

8 2. THÉORÈME II. Si pour tout point $\left(\xi, \eta_{\mathbf{1}}, \ldots, \eta_{n}\right)$ du domaine $\Omega$ et tout système $p_{1}(x), \ldots, \varphi_{n}(x)$ de fonctions continues satisfaisant dans un intervalle situé d̀ droite de $\xi$, au système d'inégalités (2) il existe un $\delta>0$ et une intégrale $\alpha_{1}(x), \ldots$ du système (1) passant par le point $\left(\xi, \eta_{1}, \ldots, \eta_{n}\right)$ telle que dans l'intervalle $\langle\xi, \xi+\delta\rangle$ aient lieu les inégalités

$$
\varphi_{i}(x) \leqslant \alpha_{i}(x) \quad(i=1,2, \ldots, n),
$$

alor.s

(I) les fonctions $f_{i}\left(x, y_{1}, \ldots, y_{n}\right) \quad(i=1,2, \ldots, n)$ sont localement croissantes au sens large par rapport d̀ chacune des variables $y_{1}, \ldots, y_{i-1}$, $y_{i+1}, \ldots, y_{n}$ séparément,

(II) les fonctions $f_{i}\left(x, y_{1}, \ldots, y_{n}\right)(i=1,2, \ldots, n)$ sont croissantes au sens large par rapport à la variable $y_{i}$ dans un voisinage suffisamment petit de tout point du domaine $\Omega$.

Remarque. On pourrait bien formuler ces deux thèses en une seule. Nous les avons séparées car cela facilitera la démonstration du théorème.

Démonstration de la thèse (I). Nous nous appuyerons sur le théorème suivant (voir [3], p. 128, lemme 2):

Si pour toute intégrale $\beta_{1}(x), \ldots, \beta_{n}(x)$ du système (1) et tout poinl $\left(\xi, \eta_{1}, \ldots, \eta_{n}\right) d u$ domaine $\Omega$ tel que $\beta_{i}(\xi) \leqslant \eta_{i}(i=1,2, \ldots, n)$ il existe une intégrale $\alpha_{1}(x), \ldots, \alpha_{n}(x)$ du système (1) passant par le point $\left(\xi, \eta_{1}, \ldots, \eta_{n}\right)$ et un $\delta>0$ tels que dans l'intervalle $\langle\xi, \xi+\delta\rangle$ aient licu les inégalités $\beta_{i}(x) \leqslant \alpha_{i}(x)(i=1,2, \ldots, n)$, alors les fonctions $f_{i}\left(x, y_{1}, \ldots, y_{n}\right)$ sont localement croissantes au sens large par rapport $\dot{a}$ chacune des variables $y_{1}, \ldots, y_{i-1}, y_{i+1}, \ldots, y_{n}$ séparément.

Prenons une intégrale quelconque $\beta_{1}(x), \ldots, \beta_{n}(x)$ du système (1) pour laquelle $\beta_{i}(\xi) \leqslant \eta_{i}$. On a

et aussi

$$
\beta_{i}(x)=\beta_{i}(\xi)+\int_{\xi}^{x} f_{i}\left(x, \beta_{1}(x), \ldots, \beta_{n}(x)\right) d x
$$

$$
\beta_{i}(x) \leqslant \eta_{i}+\int_{\xi}^{x} f_{i}\left(x, \beta_{1}(x), \ldots, \beta_{n}(x)\right) d x,
$$

c'est-à-dire l'intégrale $\beta_{1}(x), \ldots, \beta_{n}(x)$ satisfait au système d'inégalités (2). Il existe alors, en vertu de l'hypothèse de notre théorème, un $\delta>0$ et une intégrale $\alpha_{1}(x), \ldots, \alpha_{n}(x)$ passant par le point $\left(\xi, \eta_{1}, \ldots, \eta_{n}\right)$ tels que $\beta_{i}(x) \leqslant \alpha_{i}(x) \quad(i=1,2, \ldots, n)$ et $x \in\langle\xi, \xi+\delta\rangle$. L'hypothèse $d u$ Théorème II implique donc celle du théorème auxiliaire cité. Il s'ensuit que les fonctions $f_{i}\left(x, y_{1}, \ldots, y_{n}\right)$ sont croissantes au sens large par rapport à chacune des variables $y_{1}, \ldots, z$

$y_{i-1}, y_{i+1}, \ldots, y_{n}$.

Démonstration de la thèse (II). Pour la démonstration par l'absurde supposons que pour un certain indice $i$ la fonction $f_{i}\left(x, y_{1}, \ldots, y_{n}\right)$ ne soit pas localement croissante par rapport à la variable $y_{i}$; sans restreindre la généralité nous pouvons supposer que ce soit la fonction $f_{1}\left(x, y_{1}, \ldots, y_{n}\right)$. Cela veut dire que dans un voisinage suffisamment petit d'un cer-

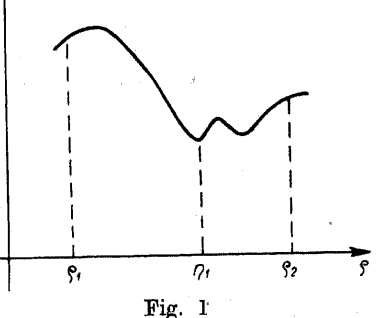
tain point $d u$ domaine $\Omega$ il existe deux points $\left(\xi, \varrho_{1}, \eta_{2}, \ldots, \eta_{n}\right),\left(\xi, \varrho_{2}, \eta_{2}, \ldots, \eta_{n}\right)\left(\varrho_{1}<\varrho_{2}\right)$ tels que

$1^{\circ}$ le segment qui les joint appartienne entièrement au domaine $\Omega$, $2^{0} f_{1}\left(\xi, \varrho_{1}, \eta_{2}, \ldots, \eta_{n}\right)>f_{1}\left(\xi, \varrho_{2}, \eta_{2}, \ldots, \eta_{n}\right)$.

Il existe alors dans l'intervalle $\left(\varrho_{1}, \varrho_{2}\right)$ (comparer le diagramme de la fonction $z=f_{1}\left(\xi, \varrho, \eta_{2}, \ldots, \eta_{n}\right)$ dans la figure 1 ) un $\eta_{1}$, tel que

$$
\left.f_{1}\left(\xi, \eta_{1}, \eta_{2}, \ldots, \eta_{n}\right)<f_{1}\left(\xi, \varrho, \eta_{2}, \ldots\right) \quad \text { pour } \quad \varrho \epsilon<\varrho_{1}, \eta_{1}\right)
$$

(il suffit de prendre comme $\eta_{1}$, la plus petite valeur de $\varrho$ qui réalise le minimum absolu de la fonction $f_{1}\left(\xi, \varrho, \eta_{2}, \ldots\right)$ envisagée dans l'intervallè $\left\langle\varrho_{1}, \varrho_{2}\right\rangle$. De la croissance (déjà démontrée) des fonctions $f_{i}\left(x, y_{1}, \ldots, y_{n}\right)$ par rapport à chacune des variables $y_{1}, \ldots, y_{i-1}, y_{i+1}, \ldots, y_{n}$ séparément il résulte que pour tout point $\mathrm{du}$ domaine $\Omega$ il existe une intégrale supérieure à droite du système (1) passant par ce point (Ważewski [3], Théorème $I$, p. 122). Désignons par $\psi_{1}(x), \ldots, \psi_{n}(x)$ l'intégrale : supérieure à droite issue du point $\left(\xi, \eta_{1}, \eta_{2}, \ldots, \eta_{n}\right)$ défini ci-dessus. Nous allons construire un système $\varphi_{1}(x), \ldots, \varphi_{n}(x)$ de fonctions continues qui satisferont aux inégalités (2), sans satisfaire pourtant aux inégalités

$$
\varphi_{i}(x) \leqslant \psi_{i}(x) \quad(i=1,2, \ldots, n)
$$

dans aucun voisinage du point $\xi$, quelque petit qu'il soit.

C'est ainsi que nous achèverons la démonstration de la thèse (II) car pour le système ainsi construit de fonctions $\varphi_{1}(x), \ldots, \varphi_{n}(x)$ l'intégrale supérieure à droite $\psi_{1}(x), \ldots, \psi_{n}(x)$ ne peut être l'intégrale intervenant dans l'hypothèse du théorème II. Mais si une intégrale 
$\alpha_{1}(x), \ldots, a_{n}(x)$ du système (1) passant par le point $\left(\xi, \eta_{1}, \ldots, \eta_{n}\right)$ satisfait aux inégalités (9)

$$
\varphi_{i}(x) \leqslant \alpha_{i}(x) \quad(i=1,2, \ldots, n ; \xi \leqslant x \leqslant \xi+\delta)
$$

l'intégrale supérieure à droite le fait aussi. Pour le système de fonctions $p_{1}(x), \ldots, \varphi_{n}(x)$ il n'existerait donc aucune intégrale du système (1) satisfaisant dans un intervalle suffisamment petit aux inégalités (9) ce qui est contraire à notre hypothèse.

\section{Construction du système de fonctions $\varphi_{1}(x), \ldots, \varphi_{n}(x)$}

Soit $K\left[\xi \leqslant x \leqslant \xi+h, \eta_{1}-h \leqslant y_{1} \leqslant \eta_{1}+h, \ldots, \eta_{n}-h \leqslant y_{n} \leqslant \eta_{n}+h\right]$, $h>0$, un parallélépipède appartenant entièrement au domaine $\Omega$. In vertu de la continuité des fonctions $f_{i}\left(x, y_{1}, \ldots, y_{n}\right)$ il existe un $M$ tel qu'on ait dans ce parallélépipède

$$
\text { (10) } \quad\left|f_{i}\left(x, y_{1}, \ldots, y_{n}\right)\right| \leqslant M \quad(i=1,2, \ldots, n ; M \geqslant 1)
$$

Dans l'intervalle $\langle\xi, \xi+h \mid M\rangle$ nous définissons les fonctions $\varphi_{2}(x)$, $\varphi_{3}(x), \ldots, \varphi_{n}(x)$ par les formules

$$
\varphi_{i}(x)=\eta_{i}-M(x-\xi) \quad(i=2,3, \ldots, n) .
$$

Nous allons démontrer que les fonctions $\varphi_{2}(x), \ldots, p_{n}(x)$ satisferont aux inégalités (2) indépendamment de la définition de la fonction $\varphi_{1}(x)$, pourva que

$$
-h \leqslant \varphi_{1}(x)-\eta_{1} \leqslant h .
$$

En effet, on peut écrire les identités (11) de la manière suivante:

$$
\varphi_{i}(x)=\eta_{i}+\int_{\xi}^{x}(-M) d x \quad(i=2,3, \ldots, n) .
$$

Mais, en vertu des définitions des fonctions $p_{2}(x), \ldots, \varphi_{n}(x)$ et de la condition (12) la courbe $y_{1}=\varphi_{1}(x), \ldots, y_{n}=\varphi_{n}(x)$ envisagéo dans l'intervalle $\langle\xi, \xi+h \mid M\rangle$ appartient au parallélépipède $K$. On a donc

$$
-M \leqslant f_{i}\left(x, \dot{\varphi}_{1}(x), \ldots, \varphi_{n}(x)\right) \quad(i=1,2, \ldots, n ; x \in\langle\xi, \xi+h / M\rangle)
$$

et par suite

$$
\varphi_{i}(x) \leqslant \eta_{i}+\int_{\xi}^{x} f_{i}\left(x, \varphi_{1}(x), \ldots, \varphi_{n}(x)\right) d x \quad(i=2,3, \ldots, n)
$$

c'est-à-dire les fonctions $\varphi_{2}(x), \ldots, \varphi_{n}(x)$ satisfont aux inégalités (2). Dans la construction de la fonction $\varphi_{1}(x)$ nous nous appuyerons sur le lemme suivant dont la démonstration sera donnée au $\S 3$ :
LEMME I. Hypothèses. Soient $G(x, y), F(x, y)$ deux fonctions définies et continues dans un domaine plan $\omega$. Soit $\left(x_{0}, y_{0}\right)$ un point de ce domaine tel que $F\left(x_{0}, y_{0}\right)=G\left(x_{0}, y_{0}\right)$ et que l'inégalité

$$
F\left(x_{0}, y_{0}\right)<G\left(x_{0}, y\right)
$$

ait lieu dans un intervalle suffisamment petit $\left\langle y_{0}-r, y_{0}\right), r>0$. Soit $T_{1}\left\langle x_{0}, x_{0}+l, y_{0}-l, y_{0}+l\right\rangle$ un rectangle appartenant au domaine $\omega$. En choisissant $M(0<M<+\infty)$ suffisamment grand on aura dans ce rectangle les inégalités

$$
|G(x, y)| \leqslant M \quad \text { et } \quad|F(x, y)| \leqslant M .
$$

Supposons de plus que $0<l<r$. Désignons par $T_{0}$ le rectangle $\left\langle x_{0}, x_{0}+h, y_{0}-l, y_{0}+l\right\rangle$ òे $h=\min [l, l / M]$. Désignons par $\psi(x)$ une intégrale arbitraire de l'équation $y^{\prime}=F(x, y)$ passant par le point $\left(x_{0}, y_{0}\right)$.

Thèse. $I l$ existe une fonction continue $\varphi(x)$ définie dans un voisinage $\grave{a}$ droite du point $x_{0}$ telle que

$$
\begin{aligned}
& \mathrm{C}_{1} \cdot \varphi\left(x_{0}\right)=y_{0} ; \\
& \mathrm{C}_{2} \cdot \varphi(x) \leqslant y_{0}+\int_{x_{0}}^{x} G(x, \varphi(x)) d x
\end{aligned}
$$

$\mathrm{C}_{3}$. son diagramme appartienne au rectangle $T_{0}$;

$\mathrm{C}_{4}$. l'inégalité $\varphi(x) \leqslant \psi(x)$ ne soit remplie dans aucun voisinage $d u$ point $x_{0}$.

A l'aide de ce lemme on peut construire la fonction cherchée $\varphi_{1}(x)$. En effet, posons

$F(x, y)=f_{1}\left(x, y, \psi_{2}(x), \ldots, \psi_{n}(x)\right), \quad G(x, y)=f_{1}\left(x, y, \varphi_{2}(x), \ldots \varphi_{n}(x)\right)$,

$$
x_{0}=\xi, \quad y_{0}=\eta_{1}, \quad \psi(x)=\psi_{1}(x) .
$$

Les hypothèses du lemme sont alors verifiées. En particulier,

$$
F\left(x_{0}, y_{0}\right)=f_{1}\left(x_{0}, y_{0}, \psi_{2}\left(x_{0}\right), \ldots\right), \quad G\left(x_{0}, y_{0}\right)=f_{1}\left(x_{0}, y_{0}, \varphi_{2}\left(x_{0}\right), \ldots\right) \text {. }
$$

Mais $\varphi_{i}\left(x_{0}\right)=q_{i}(\xi)=\eta_{i}$ et $\psi_{i}\left(x_{0}\right)=\psi_{i}(\xi)=\eta_{i}(i=2, \ldots, n)$, on a done $F\left(x_{0}, y_{0}\right)=G\left(x_{0}, y_{0}\right)$. On pent poser $\psi_{1}(x)=\psi(x)$ puisqu'on a $\psi_{1}^{\prime}(x)=$ $=f_{1}\left(x, \psi_{1}(x), \psi_{2}(x), \ldots\right)=F\left(x, \psi_{1}(x)\right)$.

En posant $\varphi_{1}(x)=\varphi(x)(\varphi(x)$ désigne la fonction intervenant dans la thèse de ce lemme) on obtient la fonction cherchée $\varphi_{1}(x)$ qui satisfait à l'inégalité

c'est-à-dire à l'inégalité

$$
\varphi_{1}(x) \leqslant \eta_{1}+\int_{\xi}^{x} G\left(x, \varphi_{1}(x)\right) d x
$$

$$
\varphi_{1}(x) \leqslant \eta_{1}+\int_{\xi}^{x} f_{1}\left(x, \varphi_{1}(x), \ldots, \varphi_{n}(x)\right) d x .
$$


En complétant par $\varphi_{\mathbf{1}}(x)$ le système déjà défini des fonctions $\varphi_{2}(x), \ldots, \varphi_{n}(x)$ on obtient le système $\varphi_{1}(x), \ldots, \varphi_{n}(x)$ satisfaisant aux inégalités $(2)$, bien que l'inégalité $\varphi_{1}(x) \leqslant \psi_{1}(x)$ ne soit remplie dans aucun voisinage du point $\xi$.

§ 3. Avant de passer à la démonstration du lemme I formulé ci-dessus nous en démontrerons un autre

LEMVE II. Soit $\varepsilon(x)$ une fonotion continue dans l'intervalle $\left\langle x_{0}, x_{0}+h\right\rangle$ telle que $\varepsilon\left(x_{0}\right)=0$ et $\varepsilon(x)>0$ pour $x_{0}<x \leqslant x_{0}+h$ (fig. 2) et soit $N$ un nombre positif. Il existe alors une suite décroissante tendant vers $x_{0}$ d'intervalles disjoints $\Delta_{1}, \Delta_{2}, \ldots$ appartenant d̀ l'intervalle $\left\langle x_{0}, x_{0}+h\right\rangle$ et tels que pour toute fonction mesurable $f(x)$ dont la valeur absolue est bornée par $N$

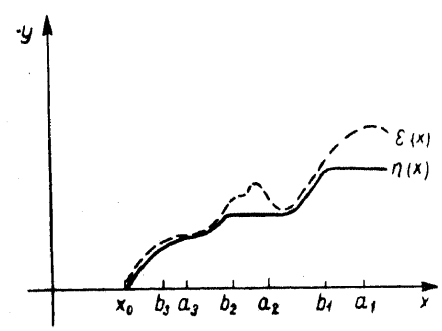
dans l'ensemble $\Sigma \Lambda_{v}$ et égale do 0 dans l'ensemble $\left\langle x_{0}, x_{0}+h\right\rangle-\sum \Delta_{v}$, on ait

$$
\left|\int_{x_{0}}^{x} f(x) d x\right| \leqslant \int_{x_{0}}^{x}|f(x)| d x \leqslant \varepsilon(x) .
$$

\section{Posons}

$$
\eta(x)=\min _{x \leqslant u \leqslant x_{0}+h} \varepsilon(u) .
$$

Fig. 2

On obtient ainsi une fonction $\eta(x)$ croissante au sens large et telle que

$$
0<\eta(x) \leqslant \varepsilon(x) \quad \text { pour } \quad x \epsilon\left(x_{0}, x_{0}+h\right\rangle ; \quad \eta\left(x_{0}\right)=0 .
$$

Choisissons arbitrairement une suite $a_{1}, a_{2}, \ldots$ décroissante, tendant vers $x_{0}$ de points de l'intervalle $\left(x_{0}, x_{0}+h\right)$ tels que $\eta\left(a_{1}\right)>\eta\left(a_{2}\right)>\ldots$ et $a_{1}=x_{0}+h$. On voit aisément que, la fonction $\eta(x)$ étant croissante, la série

$$
\sum_{\nu=1}^{\infty}\left[\eta\left(a_{v}\right)-\eta\left(a_{v+1}\right)\right]
$$

doit être convergente. Prenons maintenant $b_{v}$ entre $a_{v+1}$ et $a_{v}$ de telle manière que

$$
N\left(a_{v}-b_{v}\right)<\eta\left(a_{v+1}\right)-\eta\left(a_{v+2}\right)
$$

et posons $\Delta_{v}=\left\langle b_{v}, a_{v}\right\rangle$.

Pour tout point $x$ de l'intervalle $\left(x_{0}, x_{0}+h\right\rangle$ on peut trouver un indice $k$ tel que $a_{k+1} \leqslant x \leqslant a_{k}$. On a donc pour toute fonction mesurable $f(x)$ remplissant les conditions de notre lemme

$$
\left|\int_{x_{0}}^{x} f(x) d x\right| \leqslant \int_{x_{0}}^{x}|f(x)| d x \leqslant \sum_{\nu=k}^{\infty} \int_{b_{\nu}}^{a_{\nu}} N d x=\sum_{\nu=k}^{\infty} N\left(a_{\nu}-b_{\nu}\right) .
$$

En vertu de (16) on a donc

$$
\left|\int_{x_{0}}^{x} f(x) d x\right| \leqslant \sum_{p=k}^{\infty}\left[\eta\left(a_{v+1}\right)-\eta\left(a_{p+2}\right)\right]=\eta\left(a_{k+1}\right) \leqslant \eta(x)
$$

et par suite, en raison de (15)

$$
\left|\int_{x_{0}}^{x} f(x) d x\right| \leqslant \varepsilon(x)
$$

Démonstration du lemme I formulé au $\$ 2$. Nous démontrerons d'abord qu'en vertu des hypothèses du lemme $I$ il existe une fonction continue $\sigma(x)$ telle que

$\mathrm{C}_{5} . \sigma(x) \leqslant \psi(x)$ et $\sigma\left(x_{0}\right)=\psi\left(x_{0}\right)=y_{0} ;$

$\mathrm{C}_{6} . G(x, \sigma(x))>F(x, \psi(x))$ dans un intervalle $\left(x_{0}, x_{0}+h>\right.$;

$\mathrm{C}_{7}$. le diagramme de $\sigma(x)$ appartienne au rectangle $T_{0}$.

En effet, on a l'inégalité $F\left(x_{0}, \psi\left(x_{0}\right)\right)<G\left(x_{0}, y_{0}-l\right)$. En vertu de la continuité des fonctions $F(x, y), G(x, y)$ et $\psi(x)$ on aura l'inégalité $F(x, \psi(x))<G\left(x, y_{0}-l\right)$ dans un intervalle $\left\langle x_{0}, x_{0}+h\right\rangle$ où $h$ est une constante positive suffisamment petite.

De même il existe un rectangle $A C_{1} E_{1} D_{1}$ situé au-dessous de la courbe $y=\psi(x)$ (fig. 3) dans lequel on a $F(x, \psi(x))<$ $<G(x, y)$. Pareillement il existe un rectangle $A C_{2} E_{2} D_{2}$ (nous choisissons $D_{2}$ à gauche de $D_{1}$ ), situé au-dessous de la courbe $y=\psi(x)$, dans lequel on a la même inégalité. Construisons une suite infinie de tels rectangles de telle manière que la suite $D_{1}, D_{2}, \ldots$ tende vers $A$, que la suite $C_{1}, C_{2}, \ldots$ tende vers $B$ et qu'on ait dans chacun de

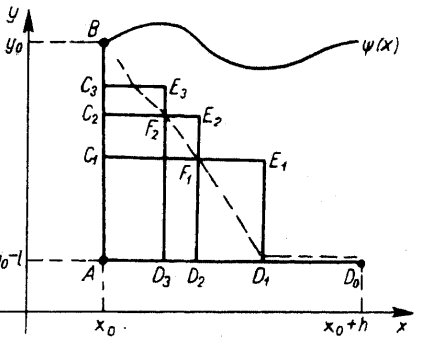

Fig. 3 ces rectangles $F(x, \psi(x))<G(x, y)$. La ligne polygonale $D_{0} D_{1} F_{1} F_{2} \ldots$ constitue le diagramme de la fonction cherchée $\sigma(x)$. Il est situé dans la somme des rectangles ainsi construits, on a donc

$$
F(x, \psi(x))<G(x, \sigma(x)) \quad \text { pour } \quad x \in\left(x_{0}, x_{0}+h\right\rangle .
$$
que

En posant $\sigma\left(x_{0}\right)=y_{0}$ on obtient, en vertu de la propriété évidente

$$
\lim _{x \rightarrow x_{0}} \sigma(x)=y_{0},
$$

la fonction $\sigma(x)$ continue dans l'intervalle $\left\langle x_{0}, x_{0}+h\right\rangle$ et satisfaisant aux conditions $\mathrm{C}_{5}-\mathrm{C}_{7}$. 
Nous avons désigné par $\psi(x)$ l'intégrale de l'équation $y^{\prime}=\mathbb{F}(x, y)$ passant par le point $\left(x_{0}, y_{0}\right)$. On a dono

En posant

$$
\psi(x)=y_{0}+\int_{x_{0}}^{x} F(x, \psi(x)) d x .
$$

$$
\lambda(x)=y_{0}+\int_{x_{0}}^{x} G(x, \sigma(x)) d x
$$

on obtient, en vertu de la condition $\mathrm{C}_{5}$ et de l'inégalité $(17), \sigma(x) \leqslant \psi(x)<$ $\left\langle\lambda(x)\right.$ pour $x \boldsymbol{\epsilon}\left(x_{0}, x_{0}+h\right\rangle$.

Posons maintenant

$$
\varepsilon(x)=\frac{1}{2}[\lambda(x)-\psi(x)] \text { pour } x \epsilon\left\langle x_{0}, x_{0}+h\right\rangle .
$$

Pour cette fonction $\varepsilon(x)$ et pour $N=2 M$ nous construisons la suite d'intervalles $A_{1}, \Delta_{2}, \ldots$ intervenant dans l'énoncé du lemme II. Définis-

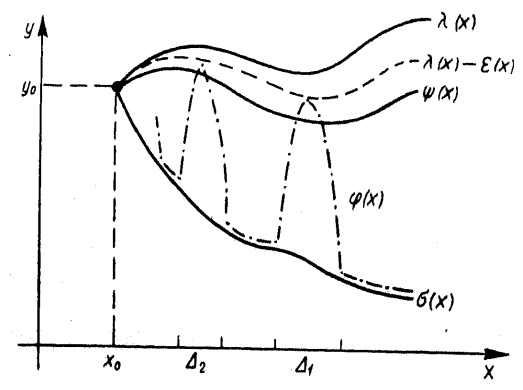

Fig. 4

sons maintenant la fonction cherchée $\varphi(x)$ de la manière suivante (fig. 4):

(a) $\varphi(x)=\sigma(x)$ dans l'ensemble $\left\langle x_{0}, x_{0}+h\right\rangle-\sum \Delta_{v}$.

( $\beta$ ) dans l'intervalle $\Delta_{v}(v=1,2, \ldots)$ posons $\varphi\left(a_{v}\right)=\sigma\left(a_{v}\right), \varphi\left(b_{v}\right)=\sigma\left(b_{v}\right)$,

$$
\varphi\left(\frac{a_{v}+b_{v}}{2}\right)=\psi\left(\frac{a_{v}+b_{v}}{2}\right)+\varepsilon\left(\frac{a_{v}+b_{v}}{2}\right) .
$$

Pour d'autres $x$ définissons $\varphi(x)$ de façon qu'elle soit continue et qu'on ait

$$
\sigma(x) \leqslant \varphi(x) \leqslant \psi(x)+\varepsilon(x),
$$

( $\gamma) \varphi\left(x_{0}\right)=y_{0}$.
On voit aisément que la fonction $\varphi(x)$ ainsi définie satisfait aux inégalités (19) dans l'intervalle $\left\langle x_{0}, x_{0}+h\right\rangle$ tout entier.

Il reste à démontrer que cette fonction $\varphi(x)$ remplit la condition $C_{2}$, c'est-à-dire qu'on a dans l'intervalle $\left\langle x_{0}, x_{0}+h\right\rangle$

$$
\varphi(x) \leqslant y_{0}+\int_{x_{0}}^{x} G(x, \varphi(x)) d x .
$$

Nous démontrerons d'abord que l'on y a l'inégalité

c'est-à-dire que

$$
y_{0}+\int_{x_{0}}^{x} G(x, \varphi(x)) d x \geqslant \lambda(x)-\varepsilon(x),
$$

$$
\int_{x_{9}}^{x}[G(x, \varphi(x))-G(x, \sigma(x))] d x \geqslant-\varepsilon(x) .
$$

Or, on a $G(x, \varphi(x))-G(x, \sigma(x)) \equiv 0$ dans l'ensemble $\left\langle x_{0}, x_{0}+h\right\rangle-\sum \Delta$ et, en raison de (14), $|G(x, \varphi(x))-G(x, \sigma(x))| \leqslant 2 M$ dans l'ensemble $\sum \Delta_{v}$. Du lemme II il résulte donc que

$$
\left|\int_{x_{0}}^{x}[G(x, \varphi(x))-G(x, \sigma(x))] d x\right| \leqslant \varepsilon(x)
$$

et, par suite, les relations (21) et (20) ont lieu.

D'autre part, en raison de (18) et (19) on a $\varphi(x) \leqslant \psi(x)+\varepsilon(x)=$ $=\lambda(x)-\varepsilon(x)$ d'où, en tenant compte de la relation (20), on obtient

$$
\varphi(x) \leqslant y_{0}+\int_{x_{0}}^{x} G(x, \varphi(x)) d x \quad \text { pour } \quad x \epsilon\left\langle x_{0}, x_{0}+h\right\rangle .
$$

La propriété $\mathrm{C}_{2}$ intervenant dans le lemme $\mathrm{I}$ se trouve ainsi établie. On peut aussi aisément démontrer que la fonction $\varphi(x)$ vérifie les propriétés $\mathrm{C}_{1}$ et $\mathrm{C}_{2}$ de ce lemme.

\section{Travaux cités}

[1] E. Kamke, Zur Theorie der Systeme gewöhnlioher Differentialgleiohungen II, Acta Mathematioa 58 (1932), p. 57-85.

[2] B. Viswanatham, On the asymptotic behaviour of the solutions of non-linear differential equations, Proc. of the Indian Acad. of Sciences. Sect. A. N 5 (1952), p. 335-341.

[3] T. Ważewski, Systèmes des équations et des inégalités différentielles ordinaires aux deuxièmes membres monotones et leurs applications, Annales de la Société Polonaise de Mathématique 23 (1950), p. 112-166. 